\title{
La standardisation continue de la langue française : l'apport des perceptions et attitudes langagières
}

Ongoing standardisation of the French language: the contribution of language perceptions and attitudes

\section{Zoë Boughton}

\section{OpenEdition}

\section{Journals}

Édition électronique

URL : https://journals.openedition.org/lbl/2183

DOI : $10.4000 / \mathrm{lbl} .2183$

ISSN : 2727-9383

\section{Éditeur}

Université de Bretagne Occidentale - UBO

\section{Édition imprimée}

Date de publication : 1 novembre 2011

Pagination : 37-57

ISBN : 978-2-901737-92-6

ISSN : 1270-2412

\section{Référence électronique}

Zoë Boughton, «La standardisation continue de la langue française : l'apport des perceptions et attitudes langagières », La Bretagne Linguistique [En ligne], 16 | 2011, mis en ligne le 01 mai 2021 consulté le 22 mai 2021. URL : http://journals.openedition.org/lbl/2183 ; DOI : https://doi.org/ $10.4000 / \mathrm{lbl} .2183$

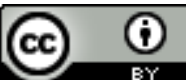

La Bretagne Linguistique est mise à disposition selon les termes de la Licence Creative Commons Attribution 4.0 International. 


\title{
La standardisation continue de la langue française : l'apport des perceptions et attitudes langagières ${ }^{1}$
}

\begin{abstract}
L
diminution de l'étendue de la variation diatopique dans le français métropolitain est attestée depuis plusieurs décennies. Mais quelle est l'interaction entre ce nivellement (ou standardisation) de la prononciation et la capacité des locuteurs à percevoir et à bien identifier les origines géographiques et sociales d'autrui ? Dans cet article, nous présentons et comparons des résultats de diverses expériences en dialectologie perceptive du français afin de mesurer la justesse de telles identifications ainsi que de montrer et d'analyser les croyances et les attitudes des informateurs envers la vitalité continuelle des accents dans le français parlé contemporain.
\end{abstract}

Nous abordons ainsi des questions plus générales de processus (observons-nous la standardisation des accents, le nivellement, la convergence ?) à travers des études plutôt qualitatives de la percep-

* Senior Lecturer in French, Department of Modern Languages, University of Exeter

1. Je tiens à remercier les organisateurs du Séminaire de la Bretagne Linguistique de leur invitation à intervenir lors de la journée d'études de décembre 2009. Le présent article est une version révisée et raccourcie de Boughton 2006. 
tion et par rapport à des études quantitatives de la production langagière, c'est-à-dire de la variation phonologique. Est-il exact de dire que la standardisation de la langue française progresse toujours? Peut-on dire que ce que l'on observe dans le français hexagonal diffère significativement de ce qui paraît être le cas dans d'autres pays, notamment la Grande-Bretagne?

Dans un article traitant des avis de locuteurs parisiens et provençaux au sujet de la variation régionale du français, Kuiper (2005) affirme que 'la perception, c'est la réalité'. Ici, nous visons donc aussi à examiner ce qui est signifié par ces termes 'perception' et 'réalité', par exemple en comparant des recherches antérieures de Kuiper (1999) avec une expérience d'identification d'accent effectuée à Rennes en Bretagne. Cette comparaison révèle une divergence intéressante entre ce que les locuteurs croient savoir de la variation dans le français, et ce qu'ils perçoivent réellement une fois confrontés à des échantillons de voix authentiques.

Dans une deuxième partie, nous traiterons la question de la capacité des auditeurs à identifier exactement les origines sociales et géographiques d'autres Français sur la seule base de leur discours en présentant quelques données qui résultent d'une autre expérience d'identification d'accents entreprise plus récemment dans la région des Pays de la Loire. En examinant les résultats, nous aborderons deux questions principales : premièrement, qu'est-ce que nous apprenons des capacités des locuteurs à percevoir et à identifier les accents des autres, et de leurs attitudes envers la variation dans le français parlé; et deuxièmement, que pouvons-nous dire quant à l'ampleur du nivellement, et le progrès de la norme, dans le français parlé urbain du nord de la France?

\section{Contexte : l'étude perceptive de Rennes}

L'étude dans les Pays de la Loire, présentée ci-dessous, était conçue comme le développement d'un projet de recherche effectué à Rennes en 1997 et 1998 (Armstrong et Boughton, 1998 ; Boughton, 2001, 2003), ce dernier étant un des éléments d'une étude plus étendue examinant l'uniformisation phonologique dans le français 
urbain septentrional (Boughton, 2003). Dans le contexte de cette étude, un grand corpus de français parlé était recueilli à Nancy (en Lorraine) et à Rennes (en Bretagne); l'âge et la stratification sociale des deux échantillons de 32 locuteurs interviewés dans chacune des deux villes (c'est-à-dire 64 locuteurs au total) sont indiqués dans le tableau 1.

Tableau 1 : Âge et structure des échantillons de locuteurs à Nancy et à Rennes

\begin{tabular}{ccccc} 
& \multicolumn{2}{c}{ HOMMES } & FEMMES \\
\hline ÂGE $/$ CLASSE & CO & CM & CO & CM \\
\hline $16-25$ & 4 JHO & 4 JHM & 4 JFO & 4 JFM \\
$40-60$ & 4 HAO & 4 HAM & 4 FAO & 4 FAM \\
\hline
\end{tabular}

Légende : JHO = jeune homme, classe ouvrière; FAM = femme plus âgée, classe moyenne, etc.

Le but principal de ces enquêtes de terrain était de déterminer l'ampleur de la standardisation phonologique dans deux villes semblables du point de vue démographique mais éloignées l'une de l'autre sur le plan géographique. Cependant, après avoir terminé l'enquête à Nancy, nous avons décidé de tester nos propres impressions de manque d'un accent localisé marqué en demandant à un groupe de locuteurs français d'écouter et de juger des échantillons représentatifs du français parlé à Nancy. Ceci a été effectué à Rennes par l'inclusion d'une épreuve auditive à la fin des interviews, qui étaient par ailleurs typiques de la méthode d'enquête sociolinguistique variationniste développée notamment par William Labov (1966/2006, 1972). Quarante informateurs rennais, socialement stratifiés selon les indications déjà montrées dans le tableau 1 , ont été invités à écouter des extraits d'environ une minute du discours spontané d'un sous-échantillon de huit des locuteurs nancéiens. Des enregistrements de ce sous-échantillon avaient été copiés sur cassette dans un ordre aléatoire et chaque extrait était représentatif d'une catégorie différente, c'est-à-dire un des jeunes hommes de la classe ouvrière, une des femmes plus âgées de la classe moyenne, etc. Après avoir écouté chaque extrait, 
les auditeurs rennais ont répondu à quatre questions qui étaient formulées de façon assez ouverte afin de ne pas trop influencer les réactions possibles aux voix. Voici les quatre questions :

A : À votre avis, est-ce que la personne qui parle a un accent?

$\mathrm{B}$ : Est-ce que la personne appartient plutôt à la classe ouvrière ou à la classe moyenne?

$\mathrm{C}$ : Est-ce que vous pouvez repérer la région d'origine de la personne?

D : Est-ce que vous trouvez leur accent/façon de parler agréable?

Puisque nous nous concentrons principalement sur l'identification régionale de l'accent, les réponses aux questions $\mathrm{A}$ et $\mathrm{C}$ sont montrées dans la figure 1.

(En résumé, la classe sociale était en général bien identifiée, et il y avait une corrélation inverse entre perception comme 'agréable' et saillance perçue de l'accent.)

Figure 1 : Réponses affirmatives à la question $\mathrm{A}$ ('oui, la personne a un accent') et identifications régionales 'exactes' des huit locuteurs nancéiens

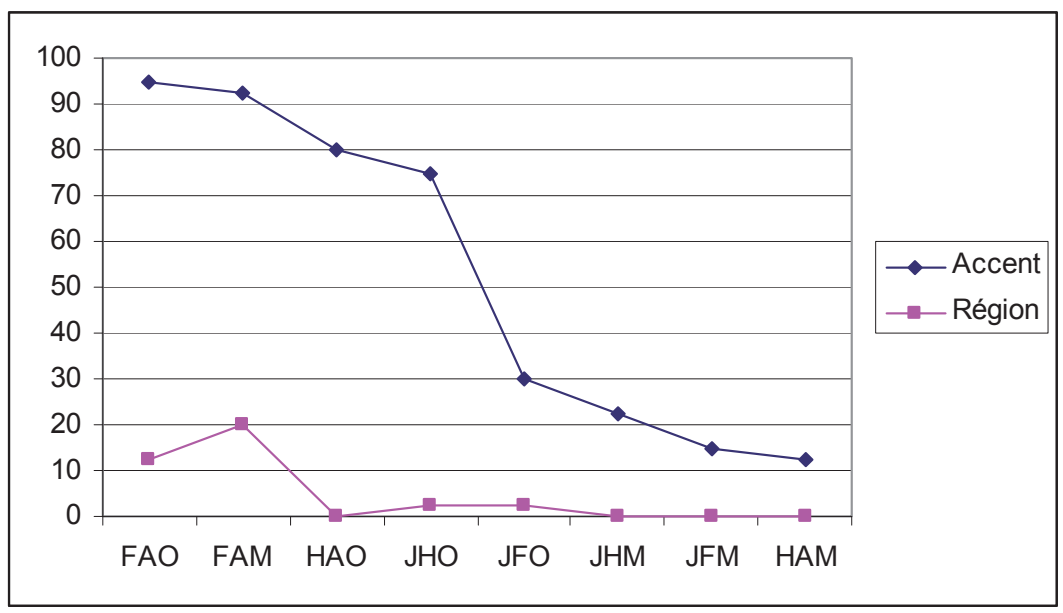


La figure 1 montre les pourcentages d'identifications régionales correctes des huit locuteurs nancéiens à côté des réponses affirmatives à la question $\mathrm{A}$, 'À votre avis, est-ce que la personne qui parle a un accent?' Les locuteurs sont rangés le long de l'axe horizontal par ordre de diminution de la saillance de l'accent perçu de gauche à droite (ou de 'accent marqué' à 'accent neutre'). Le résultat évident démontré ici est que les auditeurs rennais ne pouvaient pas pour la plupart identifier l'origine régionale du sous-échantillon de locuteurs nancéiens, d'une façon plus ou moins indépendante de la perception d'un accent quelconque. Pour la plupart des huit locuteurs, la proportion d'identifications régionales correctes reste près de zéro, malgré une interprétation plutôt généreuse de ce qui était considéré comme 'correct': si l'est de la France était mentionné dans une réponse, elle était classée comme précise, même si l'informateur devinait aussi le Québec, Paris, ou même Rennes, par exemple. La réponse la plus fréquente à la question $\mathrm{C}$ était de loin 'je ne sais pas' ou 'je n'ai aucune idée', qui représente en moyenne à peu près $61 \%$ de toutes les réponses données.

Comment ce résultat peut-il être expliqué? Nous observons que peu de traits de prononciation localisés sont présents ; et même quand il y en a, peu d'informateurs ont eu la connaissance nécessaire pour leur permettre d'indiquer exactement leur origine. En effet, l'aspect régional n'a pas été souvent mentionné en réponse aux huit voix. Ceci suggère un manque général de conscience parmi le groupe d'auditeurs de traits localisés distinctifs de prononciation dans les extraits.

Pourtant, nous pouvons contraster ce manque apparent de conscience avec des résultats obtenus par Kuiper (1999) dans une étude de dialectologie perceptive qui a employé d'autres méthodes plus 'classiques' dans ce champ (voir Preston, 1989, 1999 ; Long et Preston, 2002) pour examiner les perceptions d'un groupe de $76 \mathrm{~Pa}-$ risiens envers le français régional. Il a donné à ses informateurs une carte de la France et leur a demandé soit d'encercler soit d'indiquer par écrit toutes les régions «où les gens ont une manière particulière de parler» (Kuiper, $1999: 244)$. Une carte sommaire composée de toutes les réponses montre que l'Alsace-Lorraine termine à la 
deuxième place parmi les régions les plus souvent indiquées $(55 / 76$ ou $72 \%$ de réponses). En ceci l'Alsace-Lorraine n'a été précédée que par la Provence (63/76), et était suivie, à la troisième place, par le Nord/Lille (44/76).

Les informateurs étaient ensuite invités à classer 24 variétés régionales de français selon le degré de différence par rapport à la norme, la perception comme 'correcte' et la perception comme 'agréable'. Le français de la Lorraine a reçu un classement très haut (ou plutôt bas) sur chacun des trois critères : $20^{\mathrm{e}}$ sur 24 pour le degré de différence à la norme, $21^{\mathrm{e}}$ pour la perception comme 'correct' et $22^{\mathrm{e}}$ pour la perception comme 'agréable'. Pour ce groupe de Parisiens donc, le français de la Lorraine se situe en bas de l'échelle perceptuelle, puisqu'ils le trouvent fortement divergent par rapport à la norme, ainsi qu'incorrect et désagréable.

Par contre, les résultats de notre étude à Rennes démontrent que quand une méthode différente est adoptée - à savoir quand on présente aux informateurs des échantillons authentiques du français de Lorraine (bien que ce soit un français lorrain urbain, et de substrat roman) -, ils perçoivent très peu de divergence, et ne peuvent pas, en général, associer les voix avec une région particulière même quand ils perçoivent de la divergence. Ainsi semble-t-il qu'il existe un espace intéressant et révélateur entre ces différents types de perceptions, obtenus à l'aide de différents outils expérimentaux, aussi bien qu'entre différents types de réalités.

\section{L'étude perceptive des Pays de la Loire}

\section{Hypothèse}

La motivation initiale pour l'étude dans la région des Pays de la Loire était de développer la recherche effectuée à Rennes ainsi que de mettre en évidence les faiblesses possibles de sa méthode. L'hypothèse principale, cependant, demeurait la même : à cause du nivellement des traits de prononciation régionaux distinctifs, les auditeurs trouveront difficile la tâche d'identification exacte des origines géographiques des échantillons de variétés urbaines du français septentrional. 


\section{Méthode}

Cette fois, l'enquête de terrain était effectuée dans la région des Pays de la Loire, premièrement à La Baule (département de LoireAtlantique) et ensuite dans le département du Maine-et-Loire, en 2002-2004 par Tracy Agnew.

Le groupe d'auditeurs comptait 32 personnes, 16 hommes et 16 femmes, socialement stratifiés par âge et classe sociale comme nous avons vu dans le tableau 1. Chacun des auditeurs était originaire d'un de ces deux départements des Pays de la Loire, ou y habitait depuis au moins dix ans.

Les lieux d'enquête étaient sélectionnés pour des raisons pratiques, mais il y a également un intérêt théorique dans leur proximité relative à Rennes (environ 130 kilomètres) comparée à leur distance de Nancy (environ 750 kilomètres). L'inclusion des échantillons de voix provenant des deux villes (plutôt que de Nancy seule comme dans l'étude de Rennes) ajoute un autre élément à l'hypothèse indiquée plus tôt : si les informateurs des Pays de la Loire ne peuvent pas distinguer les locuteurs de Nancy de ceux de Rennes, ceci confirmerait l'hypothèse que la prononciation du français septentrional est fortement nivelée.

La cassette était alors conçue pour examiner cette question: 32 extraits y étaient enregistrés dans un ordre aléatoire, avec seize locuteurs de Rennes et seize de Nancy suivant les indications du tableau 2. L'échantillon de locuteurs est donc multiplié par quatre en comparaison avec le groupe de huit locuteurs de Nancy qui figuraient dans l'étude antérieure de Rennes.

Tableau 2 : Locuteurs nancéiens et rennais échantillonnés dans l'étude perceptive des Pays de la Loire

\begin{tabular}{|c|c|c|c|c|c|c|c|c|}
\hline \multirow[b]{3}{*}{ ÂGE } & \multicolumn{4}{|c|}{ NANCY } & \multicolumn{4}{|c|}{ RENNES } \\
\hline & \multicolumn{2}{|c|}{ HOMMES } & \multicolumn{2}{|c|}{ FEMMES } & \multicolumn{2}{|c|}{ HOMMES } & \multicolumn{2}{|c|}{ FEMMES } \\
\hline & $\mathrm{CO}$ & CM & $\mathrm{CO}$ & CM & $\mathrm{CO}$ & CM & $\mathrm{CO}$ & $\mathrm{CM}$ \\
\hline $16-25$ & $2 \mathrm{JHO}$ & $2 \mathrm{JHM}$ & $2 \mathrm{JFO}$ & 2 JFM & $2 \mathrm{JHO}$ & $2 \mathrm{JHM}$ & 2 JFO & $2 \mathrm{JFM}$ \\
\hline $40-60$ & $2 \mathrm{HAO}$ & 2 HAM & $2 \mathrm{FAO}$ & 2 FAM & $2 \mathrm{HAO}$ & 2 HAM & $2 \mathrm{FAO}$ & 2 FAM \\
\hline
\end{tabular}

Légende $: \mathrm{JHO}=$ jeune homme, classe ouvrière; FAM = femme plus âgée, classe moyenne, etc. 
Une autre différence par rapport à l'étude de Rennes se trouve dans le style de discours utilisé : cette fois, les extraits sont issus de lectures et non plus de passages spontanés de conversations ${ }^{2}$. Ceci assure l'uniformité de ce qui est dit, mais naturellement signifie que les locuteurs sont susceptibles de surveiller leur prononciation plus étroitement, ce qui pourrait aboutir à l'atténuation de traits localisés, s'il y en a.

Le temps moyen pris pour lire l'extrait est de 25 secondes. C'est moins que la moitié de la durée des extraits d'une minute utilisés dans l'étude de Rennes, mais néanmoins plus long que les 10-15 secondes que les informateurs de Harms (1961) ont trouvé suffisants pour leur permettre d'identifier le statut social d'autres locuteurs américains $^{3}$. Cependant, le fait que les extraits soient plus longs que ce qui semble strictement nécessaire pourrait compenser les inconvénients potentiels de l'emploi d'échantillons de lecture plutôt que de conversation spontanée.

Les informateurs ont écouté la cassette, un extrait à la fois, et ont formulé leurs jugements en réponse à ces quatre questions :

1. Pouvez-vous identifier la région d'origine de cette personne?

2. Cette façon de parler évoque-t-elle une ville particulière ou plutôt la campagne?

3. Pouvez-vous identifier cette personne en termes d'origine sociale ou de catégorie socioprofessionnelle?

4. Pouvez-vous indiquer les caractéristiques évoquées par cette façon de parler (p. ex. beau, laid, correct, accent des banlieues...)?

2. Le texte de l'extrait est comme suit (voir LEFEBVRE, 1991 : 65; cf. BOUGHTON, 2003 : 62) : Tout à l'heure, le jeune homme va prendre le train. Il part. Il ne sait guère ce qui l'attend. À la gare, sur le quai, des cantines, des sacs militaires, des valises sont entassés pêle-mêle. Il rêve à l'été dernier quand il ramassait les meules de foin. Le soleil baignait la campagne d'une lumière dorée, et çà et là, des bouquets de saules offraient une ombre au bétail.

3. Voir aussi Bauvois, 1996 (extraits de vingt secondes) et WoeHrling et Boula DE MAREÜIL, 2006 (extraits de neuf secondes). 
Dans cet article, nous allons traiter seulement les réponses à la question 1. La formulation de cette question était identique dans l'étude de Rennes, mais ici les réponses possibles ont été contraintes : on a dit aux informateurs que les locuteurs venaient de la moitié nord de la France, et dix villes ${ }^{4}$ ont été indiquées sur une carte comme exemples de lieux d'origine possibles, y compris Nancy et Rennes.

\section{Question 1 : Résultats et discussion}

Après chaque extrait, on demandait premièrement aux informateurs d'identifier la région d'origine du locuteur qu'ils venaient d'entendre (question 1). Ils ont noté différents types de réponses sur une feuille : soit villes particulières, soit régions, soit les deux à la fois. Il était donc nécessaire de classer les réponses afin de faciliter l'analyse des résultats ${ }^{5}$. Nous avons alors distingué six catégories :

- Nord (= N dans les figures 2 à 7 ; y compris les réponses de Lille, Rouen, Normandie, Picardie) ;

- Est (= E; y compris les villes de Nancy, Strasbourg et Dijon) ;

- Ouest (= W; avec réponses de Brest, Nantes, Rennes, Angers/ Anjou, Loire Atlantique, Maine-et-Loire, Sarthe, Chartres, Pays de la Loire et normal(e) - parce qu'il nous semblait qu'en disant 'normal', l'auditeur laissait entendre qu'il ne remarquait aucune différence par rapport à sa propre variété, c'est-à-dire celle du Pays de la Loire) ;

- Centre (= C; y compris Tours);

- Paris (= $\mathrm{P}$; ainsi que région parisienne) ;

- 'Ne sais pas' (=? ; indiqué par un point d'interrogation ou une case vide sur le formulaire).

4. Les dix villes étaient Brest, Nantes, Rennes, Tours, Rouen, Paris, Lille, Nancy, Dijon et Strasbourg.

5. Quand les informateurs ont donné plus d'une réponse, par exemple 'centre ou ouest', les deux ont été comptées séparément, ce qui veut dire que le nombre total de réponses varie. Ceci explique pourquoi des pourcentages plutôt que des proportions (' $\mathrm{x} / 32$ ') sont indiqués dans les figures 2 à 7 ci-dessous. 
Figure 2 : Identifications régionales des locuteurs nancéiens par les auditeurs des Pays de la Loire

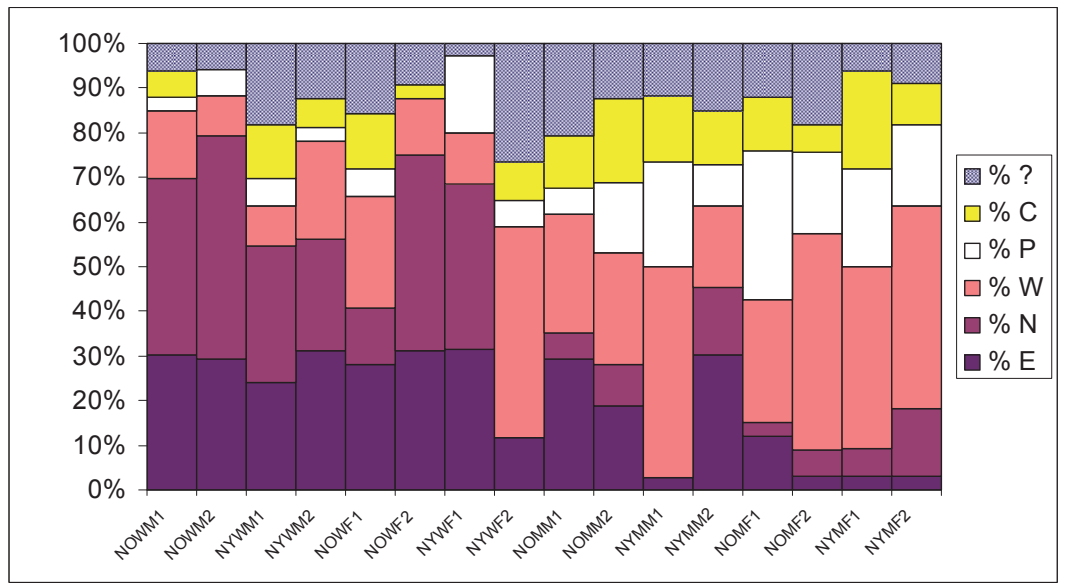

Légende : NOWM = Nancy Older Working-Class Male (Nancéien plus âgé, classe ouvrière); NYMF = Nancy Younger Middle-Class Female (jeune Nancéienne, classe moyenne), etc.

Figure 3 : Identifications régionales des locuteurs rennais par les auditeurs des Pays de la Loire

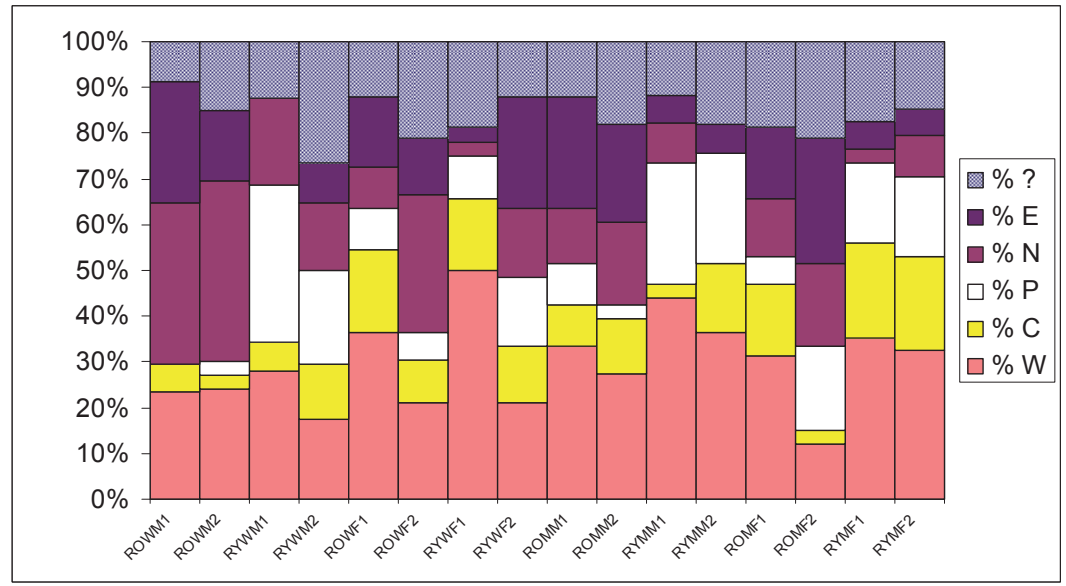

Légende : ROWM = Rennes Older Working-Class Male (Rennais plus âgé, classe ouvrière); RYMF = Rennes Younger Middle-Class Female (jeune Rennaise, classe moyenne), etc. 
Les figures 2 et 3 montrent les proportions d'identifications régionales des locuteurs réparties selon les six catégories, et divisées en deux groupes par région d'origine : Nancy en figure 2 et Rennes en figure 3. Au sein de ces deux groupes, les locuteurs sont présentés selon le même ordre de catégories sociales de gauche à droite, et cet ordre se base sur deux hypothèses de travail. Premièrement, les représentants de la même catégorie (par exemple, les hommes nancéiens plus âgés de la classe ouvrière, numérotés 1 et 2) sont mis ensemble dans l'idée qu'ils seront perçus d'une façon similaire, et deuxièmement, la position des catégories de gauche à droite reflète la supposition que l'accent devient progressivement plus neutre ou standardisé. En d'autres termes, puisque nous supposons que la catégorie socioprofessionnelle sera le facteur le plus déterminant de la divergence de la prononciation, réelle et imaginée, les locuteurs représentatifs de la classe ouvrière sont groupés à gauche, et ceux de la classe moyenne à droite. Au sein de ces groupes divisés par classe, les hommes se trouvent à gauche (moins standard) et les femmes à droite (plus standard), et au sein des groupes d'hommes et de femmes, les locuteurs plus âgés sont situés à gauche des moins âgés. Cet ordre hypothétique des locuteurs par accent du moins au plus standard se fonde et sur les conclusions générales de la recherche sociolinguistique variationniste, et sur l'affirmation peu controversée de Carton et autres (1983: 93) que le degré de standardisation de l'accent couvre un continuum dont les pôles sont âgé / rural / défavorisé / sédentaire (et masculin?) d'un côté, et jeune / urbain ou citadin / favorisé (et féminin?) de l'autre.

Pourtant, il faut remarquer que les six catégories régionales sont présentées dans un ordre différent de bas en haut dans les figures 2 et 3 : la proportion de réponses 'Est' se voit en bas des colonnes pour les locuteurs de Nancy pour faciliter la comparaison à l'intérieur de ce groupe; de la même façon, 'Ouest' se trouve en bas des colonnes pour les locuteurs rennais. Revenant aux Nancéiens, 'Nord' est audessus de 'Est' parce que cette catégorie nous semblait la deuxième plus probable entre celles qui restaient; 'Nord' est donc suivi de 'Ouest', 'Paris' et 'Centre'. Quant aux Rennais, 'Centre' et 'Paris' se situent au dessus de 'Ouest', parce que nous supposions que pour ce groupe de locuteurs, ces deux perceptions seraient plus probables que 'Nord' et 'Est', qui sont alors montrés en gris plus foncé vers le 
haut. Sur les deux figures, la proportion de 'ne sais pas' peut se voir tout en haut, et on n'y observe pas de tendance nette (Nancy, 12,7\% de '?' en moyenne; Rennes, $16,2 \%$ ).

On observe dans la figure 2 que la proportion de réponses 'Est' pour les locuteurs nancéiens diminue de gauche à droite, ce qui indique une corrélation avec la classe sociale. Sept des huit locuteurs ouvriers, situés à gauche, sont perçus d'une façon similaire, chacun ayant reçu environ $30 \%$ de réponses 'Est'. La deuxième jeune femme ouvrière (=NYWF2) semble être un locuteur de transition : à la droite de cette jeune femme, les proportions de 'Est' fluctuent, et puis se stabilisent près de zéro tout à fait à droite. Ce locuteur a reçu la plus grande proportion de 'ne sais pas' et un taux important de 'Ouest', indiquant qu'elle était difficile à localiser. En effet, elle marque un deuxième tournant, car elle fait partie du groupe de locuteurs de la classe moyenne à droite qui montrent une grande proportion de perceptions de 'Ouest', 'Paris' ou 'Centre'. Ceci pourrait faire preuve de la tendance d'annexation observée dans des études précédentes, par laquelle les auditeurs s'approprient ceux dont la façon de parler semble similaire à la leur ${ }^{6}$ comme étant originaires de la même région qu'eux-mêmes.

Le taux moyen général d'identifications 'correctes' des locuteurs nancéiens comme originaires de l'Est est de 20\%. Par comparaison à la moyenne de $5 \%$ trouvée dans l'étude antérieure effectuée à Rennes, ceci semble être une amélioration marquée, résultant peutêtre des renseignements supplémentaires donnés aux informateurs. Cependant, puisque les réponses sont groupées selon six catégories, le pur hasard donnerait une moyenne de $17 \%$ (cf. Woehrling et Boula de Mareüil, 2006). D'un plus grand intérêt est alors la relation entre classe sociale et perception de 'Est' : le taux moyen de 'Est' pour les locuteurs nancéiens ouvriers est de $27 \%$, mais n'est que $13 \%$ pour les locuteurs de la classe moyenne. Ceci présente un contraste avec les perceptions de 'Ouest': de $19 \%$ en moyenne pour les locuteurs ouvriers, augmentant jusqu'à $35 \%$ pour ceux de la classe moyenne. (La ville de Nancy, marquée sur la carte donnée aux informateurs en tant que renseignement contextuel, n'a reçu qu'un taux de $4,2 \%$ de réponses en moyenne.)

6. C'est-à-dire qu'ils n'observent aucune divergence saillante. 
La figure 3 montre des tendances différentes. Le taux moyen général de perceptions 'exactes' des locuteurs rennais comme originaires de 'l'Ouest' est de $30 \%$, quelque peu plus élevé que pour les Nancéiens (bien que le taux moyen de réponses précisément de Rennes ne soit que de 4,3\%). Ce résultat démontre que les auditeurs originaires du nord-ouest ont pu faire la différence entre les Rennais de l'ouest et les Nancéiens de l'est dans une certaine mesure, mais non pas de façon simple. La plus grande proportion globale d'identifications exactes pourrait renvoyer simplement à l'annexation ${ }^{7}$ et en outre, les proportions de réponses dans les autres catégories révèlent une image complexe. Nous voyons encore quelques interactions entre identification 'exacte' et classe sociale, mais dans le sens contraire à celles observées pour les locuteurs nancéiens : le taux moyen de 'Ouest' pour les locuteurs ouvriers, situés à gauche, est de $28 \%$, tandis qu'il est de $32 \%$ pour ceux de la classe moyenne, c'est-à-dire une modeste tendance à la hausse. Les perceptions de 'Est' présentent un deuxième contraste avec ce que nous avons observé pour les locuteurs de Nancy. Le taux moyen de perception des locuteurs rennais de la classe ouvrière comme étant de ' 1 'Est' est de $13 \%$, et de ceux de la classe moyenne, de $14 \%$; il $\mathrm{y}$ a donc très peu de différence quant aux groupes divisés par classe sociale. Il existe pourtant un contraste important par rapport à la classe quant aux perceptions de 'Nord' : la moyenne est de $21 \%$ pour les Rennais ouvriers, mais celle-ci tombe sensiblement à $10 \%$ pour le groupe de la classe moyenne. Nous voyons là un écart intéressant entre les Rennais divisés par classe sociale en ce qui concerne la perception d'être du Nord.

Une autre observation intéressante qu'on pourrait faire par rapport à la figure 3 concerne les parties blanches importantes pour les locuteurs ayant des taux relativement élevés de réponses de 'Paris' : à savoir, les jeunes hommes rennais. En regardant les remarques des informateurs de plus près, nous avons trouvé que les connotations de 'Paris' ne sont pas les mêmes pour ces deux groupes: pour les jeunes hommes ouvriers, une réponse de 'Paris' indique la banlieue parisienne, tandis que pour les jeunes hommes de

7. Ce qui implique que les accents de ce groupe sont moins divergents en général ; voir Boughton, 2005, pour quelques preuves empiriques corroborantes. 
la classe moyenne, 'Paris' signifie la ville, le français plus standard, probablement l'ascension sociale et la mobilité géographique et peut-être aussi l'accent 'branché' des médias. Ce qui est clair est que l'étiquette 'Paris' possède une signification sociale double, et donc pour ces jeunes hommes rennais, la divergence de l'accent est associée non pas à une différence sur le plan régional mais à des différences sociales.

Figure 4 : Graphique 'radar' représentant les identifications régionales des locuteurs nancéiens de classe ouvrière

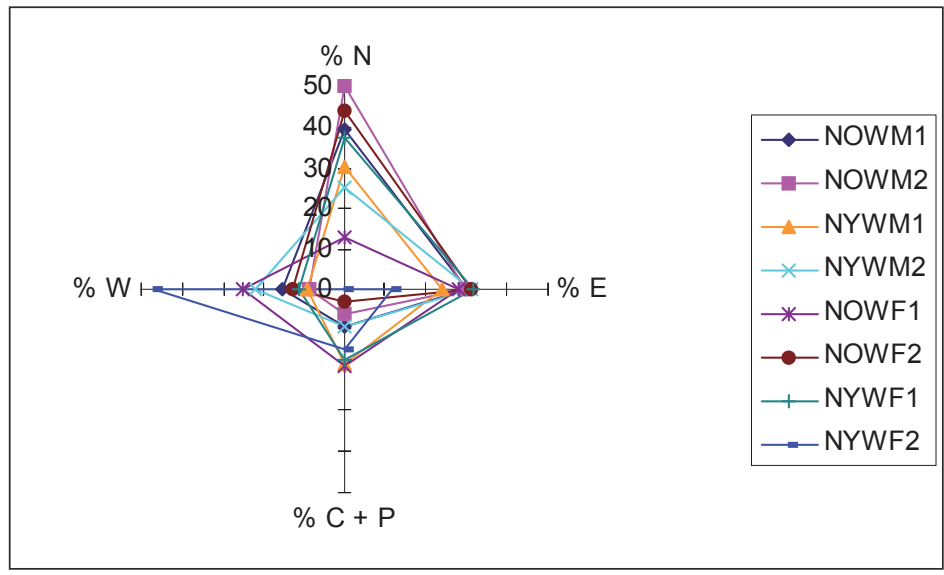

Figure 5 : Graphique 'radar' représentant les identifications régionales des locuteurs nancéiens de classe moyenne

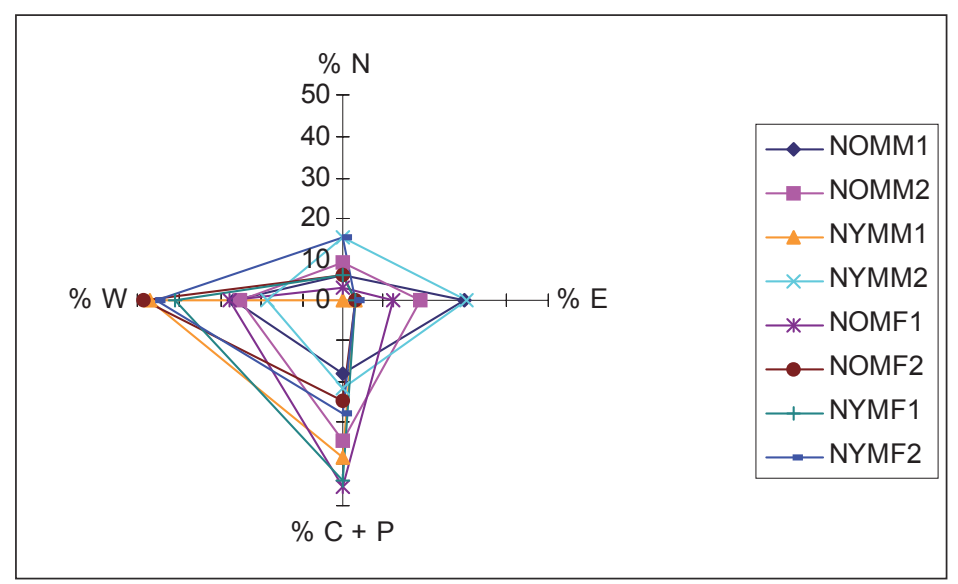


Figure 6 : Graphique 'radar' représentant les identifications régionales des locuteurs rennais de classe ouvrière

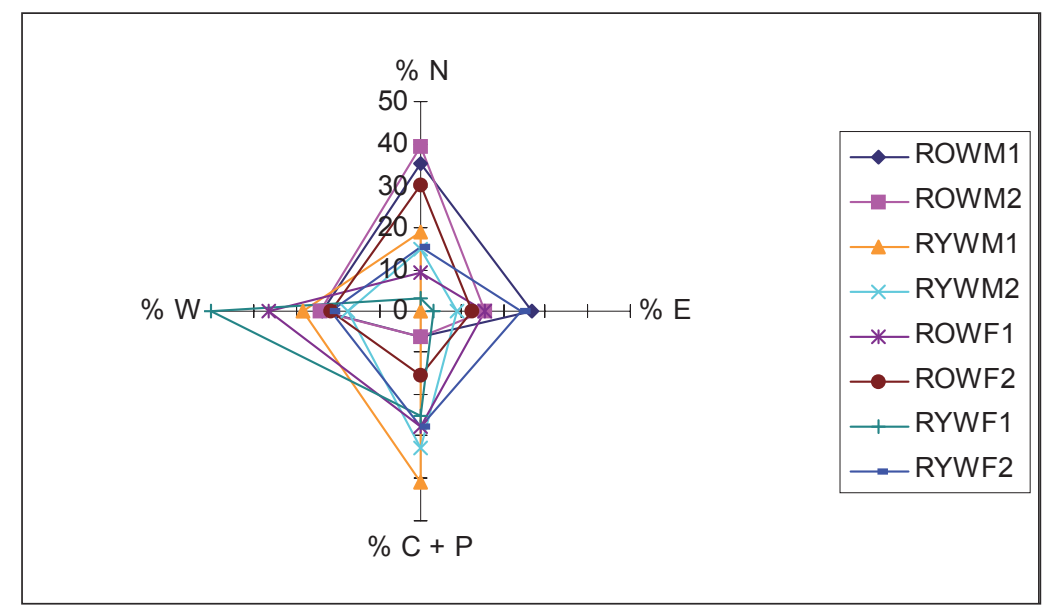

Figure 7 : Graphique 'radar' représentant les identifications régionales des locuteurs rennais de classe moyenne

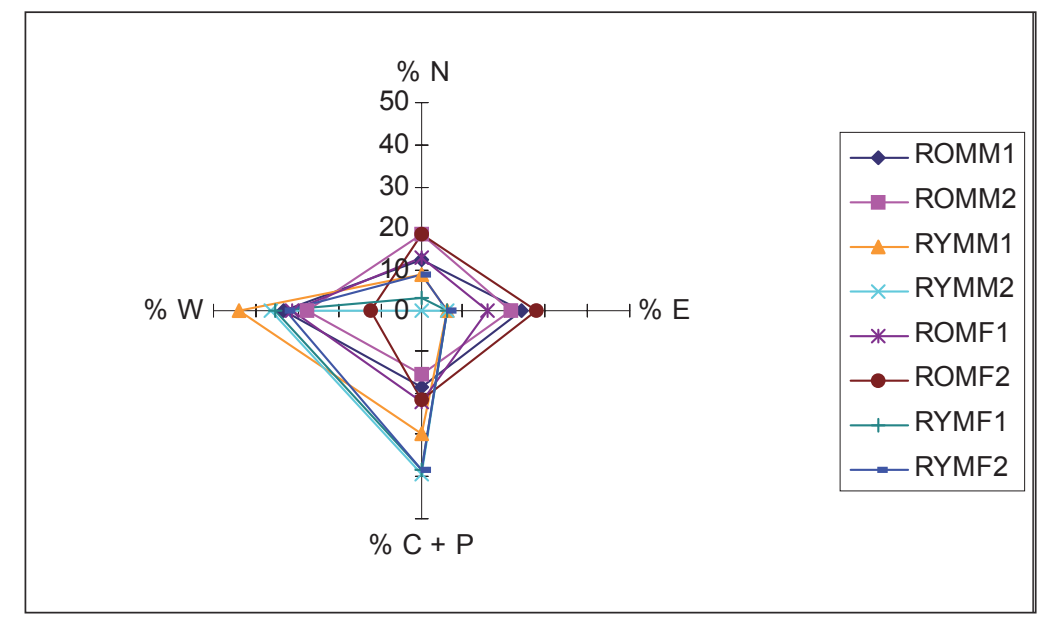


Les figures 4 à 7 montrent encore une fois les réponses à la question 1, mais ici par des graphiques de type 'radar'. Ceux-ci nous offrent la présentation visuelle peut-être la plus claire des perceptions régionales des groupes de locuteurs car on peut régler et étiqueter les axes pour refléter les quatre points cardinaux traditionnels. Au sein de chaque 'toile' radar, une ligne représente un seul locuteur et sert à joindre les points sur les axes qui sont placés selon le pourcentage d'une réponse particulière (par exemple, 'Nord') pour ce locuteur. Dans ces figures, les réponses de 'Centre' et 'Paris' ont été additionnées, d'autant plus que les deux semblent en général évoquer un manque de divergence, pour créer quatre 'directions' perceptives générales.

Les deux groupes régionaux de locuteurs sont encore une fois séparés et puis subdivisés par classe sociale: classe ouvrière en haut et classe moyenne en bas. Une comparaison des figures 4 et 5 (Nancy) avec les figures 6 et 7 (Rennes) montre des tendances similaires, mais pas identiques. Ceci implique que les auditeurs ont pu cerner des différences générales entre les groupes régionaux. En outre, au sein des groupes régionaux, les tendances observées par rapport aux classes sociales ne sont pas tout à fait les mêmes.

Quant à Nancy, les figures 4 et 5 sont presque des images inversées, ce qui souligne une différence nette liée à la classe sociale. La figure 4 (classe ouvrière) montre un effet d'entassement ou regroupement pour 'Est' ainsi qu'un fort attrait vers 'Nord' qui n'est pas observé pour les locuteurs de la classe moyenne (figure 5); inversement, la figure 5 montre que les locuteurs nancéiens de la classe moyenne sont fortement associés avec 'Ouest', 'Centre' et 'Paris', moins associés avec 'Est' et très peu avec 'Nord'. C'est alors l'axe 'Nord' qui divise le plus les classes sociales. L'exception est encore une fois la jeune femme ouvrière 'de transition' (NYWF2) dont la ligne se distingue des autres dans la figure 4, sa forme étant beaucoup plus similaire à celles montrées dans la figure 5 .

En ce qui concerne Rennes, les figures 6 et 7 démontrent encore une différence générale dans les perceptions des deux classes sociales, mais ici le contraste n'est pas aussi saisissant que pour les groupes par classe de Nancy. Ceci peut-être en raison du fait que 
l'élément régional de leur accent, s'il y en a un (voir Boughton, 2005), n'est pas aussi divergent que chez les locuteurs de Nancy et donc ne sert pas d'indice perceptif supplémentaire pour différencier les classes sociales. Les deux groupes de classe rennais montrent des perceptions similaires, quoique mixtes, de 'Est'; le radar de la classe moyenne montre un attrait plus fort et un regroupement vers 'Ouest' et 'Centre' / 'Paris', bien que plusieurs locuteurs ouvriers soient perçus d'une façon similaire et, qui plus est, les locuteurs nancéiens de classe moyenne sont plus fortement associés aux axes 'standard' de 'Ouest' et 'Centre' / 'Paris' que ceux de Rennes. Ce qui divise le plus les groupes sociaux rennais, c'est la forte association des locuteurs ouvriers avec 'Nord', une catégorie qui comprend les réponses de Lille, Rouen, Normandie et Picardie, qui se trouvent toutes à une distance importante de Rennes.

\section{Conclusion}

Les résultats présentés ci-dessus démontrent que bien que les informateurs des Pays de la Loire n'ont pas très bien réussi à identifier exactement les régions d'origine des échantillons, ils ont néanmoins pu déceler quelques différences entre les locuteurs de Nancy et ceux de Rennes. Ceci est assez impressionnant étant donné la nature des stimuli - 32 extraits de lecture identiques de 25 secondes dans un ordre aléatoire - et suggère peut-être que l'effet du style de discours échantillonné a relativement peu d'importance (observé également par Woehrling et Boula de Mareüil, 2006). Cependant, nous avons vu aussi de claires preuves d'annexation incorrecte (voir par exemple le taux de 'Ouest' pour Nancy) quand peu, ou aucune, divergence n'a été perçue, et de la dépendance déplacée vis-à-vis de stéréotypes dépassés (par exemple, que 'Nord' signifie 'classe ouvrière') quand les auditeurs ont remarqué quelques différences d'accent. Dans l'ensemble, les résultats suggèrent deux axes régionaux-perceptifs majeurs : 'Nord / Est' évoquant une divergence d'accent régionalesociale, et 'Ouest / Centre / Paris' lié à la convergence et donc à la neutralité d'accent (standardisé).

Tout au long de cette étude, nous avons vu des écarts entre des réalités empiriques (comme par exemple l'origine géographique réelle des locuteurs) et des représentations fondées au moins en partie 
sur des stéréotypes sociaux (cf. Davies, 1994; Hauchecorne et Ball, 1997). Dans quelle mesure peut-on donc affirmer que 'la perception, c'est la réalité'? Kuiper (2005 : 28) remarque que les perceptions populaires ou 'folk', c'est-à-dire des non-linguistes (Niedzielski et Preston, 2000), peuvent avoir 'peu de fondement dans la réalité empirique', mais sont néanmoins réelles psychologiquement et peuvent alors avoir un impact considérable sur les attitudes langagières, la sécurité et l'insécurité linguistique, peut-être alors sur le comportement linguistique et même la constitution de la norme. Nous pouvons ajouter que l'utilisation de stimuli authentiques, à savoir d'extraits de voix, dans la recherche perceptive peut non seulement dévoiler des écarts entre des degrés de divergence linguistique réelle et imaginée, mais pourrait aussi servir à ébranler des stéréotypes potentiellement nuisibles.

Bon nombre des informateurs ne l'ont simplement pas cru quand on leur a dit après l'épreuve auditive que les locuteurs étaient originaires de Nancy et de Rennes, et il a fallu du temps pour persuader certains d'entre eux, parfois jusqu'à trente minutes. Comme l'a dit un des auditeurs, 'on ne peut pas résumer la personnalité, l'origine d'une personne avec la voix, juste avec son timbre de voix ; peut-être il y a cinquante ans mais plus maintenant'.

De ce point de vue, quelques-uns des écarts entre les réalités empiriques et perceptives dont nous avons discuté ici nous révèlent une partie du passé de la langue, mais d'autres, tels que les différences dans les perceptions des locuteurs plus âgés et celles des jeunes, peuvent donner des indications de sa trajectoire future.

\section{Lien perception-production?}

$\mathrm{Au}$ cours de nos recherches, nous avons également étudié le comportement langagier des locuteurs dont nous avons vu les perceptions ci-dessus, un aspect particulier étant la variation dans la production des groupes finaux consonne + liquide (par exemple table $>$ tab', quatre $>$ quat'). La figure 8 montre les taux d'élision de ces liquides trouvés pour les huit locuteurs de Nancy qui figuraient dans l'étude perceptive de Rennes, ainsi que les taux de perception de ces mêmes locuteurs comme étant d'origine ouvrière. 
Figure 8 : Perception d'origine ouvrière et taux d'élision de liquides en groupes finaux des huit locuteurs nancéiens de l'étude perceptive de Rennes

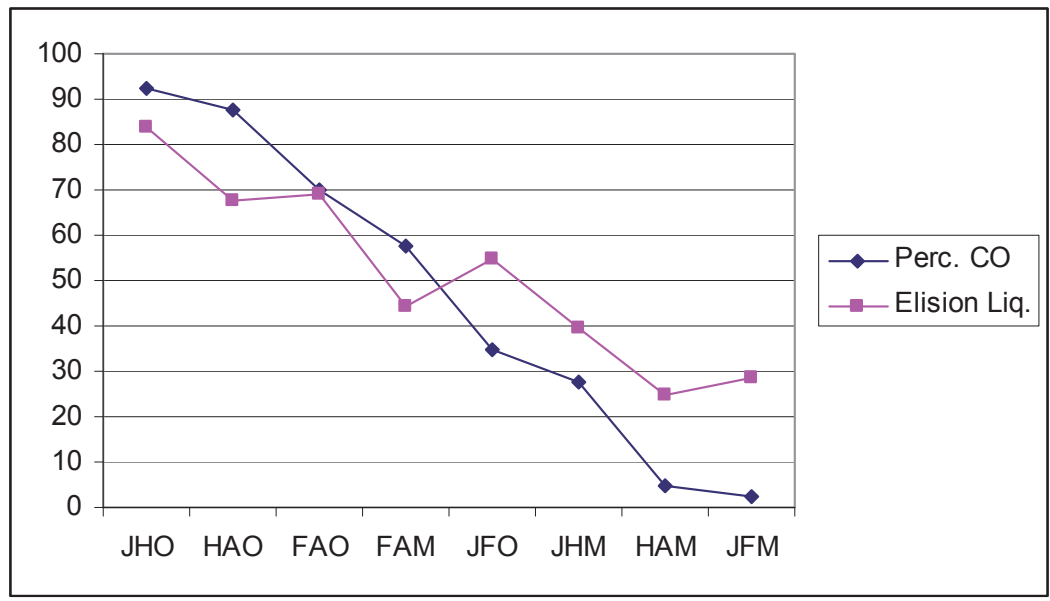

Ce que l'on peut souligner ici est que même si on observe le nivellement des accents sur le plan diatopique, de tels résultats laissent voir que les différences sur le plan diastratique peuvent néanmoins persister et, en outre, pourraient avoir une fonction diagnostique importante dans la perception de la variation sociolinguistique.

\section{Bibliographie}

ARMSTRONG Nigel et BOUGHTON Zoë, «Identification and evaluation responses to a French accent: some results and issues of methodology », Revue PArole, 5/6, 1998, p. 27-60.

Bauvois Cécile, «Parle-moi, et je te dirai peut-être d'où tu es», Revue de Phonétique Appliquée, 121, 1996, p. 291-309.

Boughton Zoë, «Methodological approaches to the study of French accent identification », dans M.-A. HinTZE, T. POOLEY et A. JUDGE éd., French Accents: Phonological and Sociolinguistic Perspectives, London, AFLS/CILT, 2001, p. 218-239. 
Boughton Zoë, Phonological variation in contemporary standard French: a tale of two cities, thèse de doctorat, University of Newcastle upon Tyne, 2003.

BOUGHTON Zoë, «Accent levelling and accent localisation in northern

French: comparing Nancy and Rennes», Journal of French Language Studies, 15, 2005, p. 235-256.

BOUGHTON Zoë, «When perception isn't reality: accent identification and perceptual dialectology in French», Journal of French Language Studies, 16, 2006, p. 277-304.

CARTON Fernand, Rossi Mario, AUTESSERRE Denis et LÉON Pierre, Les Accents des Français, Paris, Hachette, 1983.

DAVIES Winifred, «Dialect and standard in speakers' perceptions: delayed reactions to linguistic reality in a German city», dans M. PARRY, W. DAVIES et R. TEMPLE éd., The Changing Voices of Europe, Cardiff, UWP/MHRA, 1994, p. 295-309.

HARMS Leroy, «Listener judgements of status cues in speech», Quarterly Journal of Speech, 47, 1961, p. 164-168.

HAUCHECORNE Fabrice et BALL Rodney, "L'accent du Havre: un exemple de mythe linguistique», Langage et Société, 82, 1997, p. 5-25.

KUIPER Laurence, «Variation and the norm: Parisian perceptions of regional French», dans D. PRESTON éd., Handbook of Perceptual Dialectology, tome 1, Amsterdam, John Benjamins, 1999, p. 243262.

KUIPER Laurence, «Perception is reality: Parisian and Provençal perceptions of regional varieties of French ", Journal of Sociolinguistics, 9/1, 2005, p. 28-52.

LABOV William, The Social Stratification of English in New York City,

Washington D.C., Center for Applied Linguistics, 1966 / 2e édition, Cambridge, Cambridge University Press, 2006.

Labov William, Sociolinguistic Patterns, Philadelphia, University of Pennsylvania Press, 1972.

LEFEBVRE Anne, Le français de la région lilloise, Paris, Publications de la Sorbonne, 1991.

LONG Daniel et PRESTON Dennis éd., Handbook of Perceptual Dialectology, tome 2, Amsterdam, John Benjamins, 2002.

NiedZIELSKI Nancy et Preston Dennis, Folk Linguistics, Berlin, Mouton de Gruyter, 2000.

PRESTON Dennis, Perceptual Dialectology, Dordrecht, Foris, 1989.

PRESTON Dennis, «A language attitude approach to the perception of 
regional variety», dans D. PRESTON éd., Handbook of Perceptual Dialectology, tome 1, Amsterdam, John Benjamins, 1999, p. 359373.

Woehrling Cécile et Boula DE MAReÜIL Philippe, «Identification d'accents régionaux en français : perception et catégorisation», Bulletin PFC, 6, 2006, p. 89-103. 
